20th International Conference on Knowledge Based and Intelligent Information and Engineering Systems, KES2016, 5-7 September 2016, York, United Kingdom

\title{
Automatic EEG processing for the early diagnosis of Traumatic Brain Injury
}

\author{
Bruno Albert ${ }^{\mathrm{a}}$, Jingjing Zhang ${ }^{\mathrm{a}}$, Alexandre Noyvirt ${ }^{\mathrm{b}}$, Rossitza Setchi ${ }^{\mathrm{a}^{*}}$, Haldor Sjaaheim ${ }^{\mathrm{b}}$, \\ Svetla Velikova ${ }^{\mathrm{b}}$, Frode Strisland ${ }^{\mathrm{c}}$ \\ ${ }^{a}$ School of Engineering, Cardiff University, Cardiff CF24 3AA, UK, Applied Automation, Bridgend, UK, ${ }^{c}$ Smartbrain, Oslo, ${ }^{d}$ SINTEF ICT, Oslo \\ Norway
}

\begin{abstract}
Traumatic Brain Injury (TBI) is recognized as an important cause of death and disabilities after an accident. The availability a tool for the early diagnosis of brain dysfunctions could greatly improve the quality of life of people affected by TBI and even prevent deaths. The contribution of the paper is a process including several methods for the automatic processing of electroencephalography (EEG) data, in order to provide a fast and reliable diagnosis of TBI. Integrated in a portable decision support system called EmerEEG, the TBI diagnosis is obtained using discriminant analysis based on quantitative EEG (qEEG) features extracted from data recordings after the automatic removal of artifacts. The proposed algorithm computes the TBI diagnosis on the basis of a model extracted from clinically-labelled EEG records. The system evaluations have confirmed the speed and reliability of the processing algorithms as well as the system's ability to deliver accurate diagnosis. The developed algorithms have achieved $79.1 \%$ accuracy in removing artifacts, and $87.85 \%$ accuracy in TBI diagnosis. Therefore, the developed system enables a short response time in emergency situations and provides a tool the emergency services could base their decision upon, thus preventing possibly miss-diagnosed injuries.
\end{abstract}

(C) 2016 The Authors. Published by Elsevier B.V. This is an open access article under the CC BY-NC-ND license

(http://creativecommons.org/licenses/by-nc-nd/4.0/).

Peer-review under responsibility of KES International

Keywords: Artifact removal, Diagnosis; Electroencephalography (EEG); Portable Medical System; Traumatic Brain Injury (TBI).

* Corresponding author. E-mail address: setchi@cf.ac.uk

\section{Introduction}

Traumatic brain injury (TBI) is caused by an external force that damages the brain. This brain dysfunction results as possible physical, cognitive, social, emotional, and behavioral effects on the subject ${ }^{1}$. The severity of the injury 
ranges from mild to severe as well as the associated impacts on the quality of life of the person with $\mathrm{TBI}^{2,3,4,5}$. TBI has been recognized as an important cause of death in the $\mathrm{US}^{6}$ as well as in Europe ${ }^{7}$. Moreover, it leads to a great economic burden ${ }^{7}$.

Irreversible brain damages can result from a trauma that is not properly diagnosed, or too late. Hence there is a need for a reliable tool that can be used by emergency services in order to obtain a quick diagnosis of TBI at the place of injury. However, current methods and devices that provide TBI diagnosis are limited to clinical environments. In particular, contrary to other medical imagery technologies, Electroencephalography (EEG) techniques have the potential for being used in a portable way. In addition, Quantitative Electroencephalography (qEEG) is a sensitive diagnostic method of brain injury after mild head injury. It has shown over $80 \%$ accuracy in discriminating between normal and traumatic brain-injured subjects ${ }^{2,3,4}$.

The EmerEEG project addresses this problem by proposing a portable decision support system based on EEG technology for early diagnosis of TBI at the point of need. This system includes a head device for fast and simple acquisition of EEG data during emergencies, as well as necessary devices enabling processing power, interfacing and communication capabilities. This paper focuses on the processing part of the system, which, once integrated to the rest of the system, provides a tool for the automatic diagnosis of TBI and decision support. The idea is to enable anyone from the emergency services with minimal training to assess the severity of a brain injury.

The remainder of the paper is organized as follows. Related processing and diagnostic techniques, are reviewed in section 2. Section 3 outlines the EEG processing method and TBI diagnosis. Section 4 describes the evaluation of the system in terms of the quality of the EEG pre-processing and TBI diagnostics. Finally, section 5 summarizes the paper and highlights future work.

\section{Literature review}

This section reviews methods for EEG data processing and TBI diagnosis.

The clinical criterion most widely used to classify TBI severity is the Glasgow Coma Scale (GCS), which grades the condition of a patient on a scale from 3 to 15 based on verbal, motor, and eye reactions to stimuli ${ }^{8,9}$. However, the GCS is a qualitative method of assessment, which has its limitations. Advanced neuroimaging techniques like Computed Tomography (CT) and Magnetic Resonance Imaging (MRI) are now widely used in hospitals for the assessment of neurological damage. The size and non-portability of the equipment, in addition to their limitations in diagnosing mild $\mathrm{TBI}^{10,11,12}$, however, constrain their use in portable systems. By comparison, the EEG technique provides a direct measurement of brain activity without the need for external radiation or injected substances.

Rather than only analyzing raw recordings through visual inspection, the extraction of quantitative EEG data, such as frequency and coherence, has shown its relevance in recent years in supplying relevant and re-producible features for the development of diagnostic tools ${ }^{13}$. The discriminant accuracy of qEEG is reported as $95.67 \%$ in the detection of mild head injury ${ }^{3}$ and $75.8 \%$ in predicting the outcome one year after the injury ${ }^{14}$. Moreover, qEEG demonstrates $96.39 \%$ classification accuracy, $95.45 \%$ sensitivity and $97.44 \%$ specificity in discriminating between groups with mild and severe $\mathrm{TBI}^{4}$. The EEG discriminant score is also used to measure intermediate severity in moderate TBI patients. Significant correlations between EEG discriminant scores, emergency admission measures, and post-trauma neuropsychological test scores have validated the discriminant function as an index of severity of injury and a classifier of the extremes of severity ${ }^{4}$.

The procedure for computing a TBI diagnosis using EEG data normally involves pre-processing the raw recording to reduce the impact of the low signal-to-noise ratio and to obtain a more accurate representation of the pure brain activity. Artifacts are the most important cause of noise once errors directly due to the instrumentation have been eliminated. Artifacts are electrical signals detected along the scalp that do not arise from the cerebra. Typical artifacts include electrocardiography (ECG) artifacts caused by heart beats ${ }^{15}$, ocular artifacts (EOG) caused by eye blinks or low-frequency patterns caused by eye movements ${ }^{16,17}$, and muscle activity (EMG) caused by movements of the head, body, jaws, or tongue. EOG and EMG activities are unavoidable in EEG recording ${ }^{16,17,18}$. Conventional clinical approaches reduce noise by discarding epochs with artifacts through visual inspection by specialists. This manual process is time-consuming and subject to intra-observation differences, and useful information of the brain activity embedded in the discarded epochs might be lost. 
An effective and popular alternative is the use of Independent Component Analysis (ICA), which separates the artifacts from the EEG signals without removing epochs ${ }^{19,20}$. However, components corresponding to the artifacts have to be carefully selected for this method to be effective. Therefore, the key to achieving automatic artifact removal is to find a method that automatically selects artifact components from the brain activity after separation with ICA. Spatial, spectral, temporal, and statistical features have been combined to identify artifacts ${ }^{15,21,22,23,24}$. An alternative approach to automatic artifact removal is a novel technique, named Automatic Wavelet Independent Component Analysis (AWICA) ${ }^{25}$. It combines wavelet transform and ICA based on the estimation of kurtosis and Renyi's entropy. This is done in a two-step procedure, instead of applying wavelet analysis after ICA ${ }^{26}$. One important advantage of this method is that it suppresses artifact components while reducing the loss of residual informative data, since the components related to relevant EEG activity are mostly preserved ${ }^{25}$.

\section{EEG processing and TBI diagnosis}

\subsection{Offline and online operation}

This section describes the proposed algorithms for automatic detection of traumatic brain injury. The algorithms are based on data signal processing techniques and a classification approach. The aim is to alert the local operator and the remote telemedicine personnel when TBI is detected. The flowchart in Fig. 1 shows the processes for computing the TBI diagnosis online and for training the model offline using pre-recorded data. The two processes share the same pre-processing and qEEG feature extraction steps. The offline process performs these steps for all recordings from the clinical database and constructs a model using machine learning, whereas the online process applies these steps on the continuous recording coming from the portable sub-system and extracts a predicted

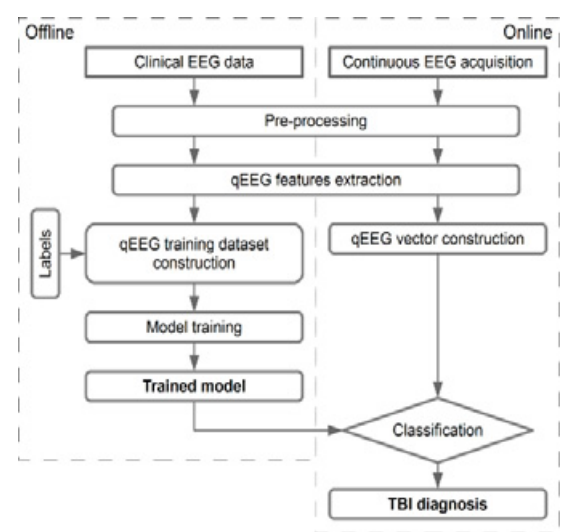

diagnosis from the trained model.

Fig. 1. Offline model training and online EEG processing and TBI diagnosis.

The continuous EEG acquisition and online processing starts after the montage of the electrodes on the patient's head is completed and the electrical contact is assured. The raw EEG recording is first pre-processed by filtering high frequency noise and removing artifacts. Next, qEEG features within four frequency bands are calculated from this 'clean' recording. In particular, 16 features that have been proven discriminant in the detection of $\mathrm{TBI}^{3}$ are used in the diagnosis step. A classification prediction is performed using discriminant analysis and the model extracted from the previously recorded data. Detailed information about data processing and classification methods is given in the following sections for both online and offline operations.

\subsection{Continuous EEG acquisition}

Clinical best practice recommends the use of at least 60 seconds of artifact-free $\mathrm{EEG}^{27,28}$. This has been confirmed by a systematic analysis using the EmerEEG system, which has shown that one minute long epochs are 
sufficient for obtaining reliable diagnosis results. Files containing segments of one minute EEG data are stored according to the European Data Format (EDF) standard ${ }^{29}$ and loaded for processing. The diagnosis process starts when the first segment of one minute EEG data is collected. In addition to montage verifications, a fault alert mechanism in the processing algorithm detects faults in signals for each channel to ensure that the diagnosis is based on reliable data. The electrodes are positioned following the 10-20 rule employed in EEG best practice and the montage used here is linked-ear.

Consider, as an example, the piece of raw EEG data shown in Fig. 2. According to the annotations made by a clinical specialist, this one minute segment contains eye blinking and electrode movement artifacts. Eye blinking artifacts mainly appear around 1, 24, 37, 43, and 56 seconds. Electrode movements occur around 2, 12, 40, and 50 seconds. These artifacts have higher amplitude and frequency compared to the brain signals. This points to the need for a pre-processing method. The next section describes the algorithm employed to remove automatically such artifacts.

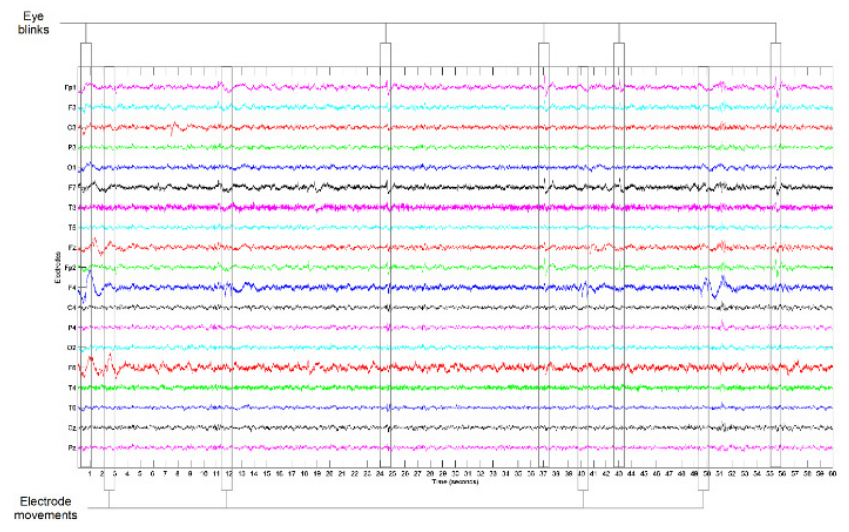

Fig. 2. Example of a one minute segment of raw EEG data.

\subsection{EEG Pre-processing}

In EEG data, the signal-to-noise ratio is usually low and the noise frequency and amplitude are higher than the brain signals. On the other hand, since the analysis of the frequency bands in the range between $0 \mathrm{and} 30 \mathrm{~Hz}$ can be sufficient for detection of $\mathrm{TBI}^{3,4}$, the recording is first filtered by a low pass filter with high limit of $30 \mathrm{~Hz}$.

The next step is the removal of artifacts, including those resulting from eye blinks, eye movements, electrode movements, muscle activity, drowsiness, and head movements. The method adopted for performing the automatic removal of artifact is the Automatic Wavelet Independent Component Analysis (AWICA) ${ }^{25}$. The flowchart of the algorithm $^{21,25}$ is shown in Fig. 3 .

The algorithm consists of five steps:

(1) Wavelet component (WC) extraction. Each channel of the filtered recording is divided into four frequency bands (delta, theta, alpha and beta) using a four-level Discrete Wavelet Transform (DWT). Each band of each channel is represented using a Wavelet Component.

(2) Critical WC selection. WCs corresponding to artifacts are automatically identified through a quantitative measure as critical WCs. The selection is based on kurtosis and Renyi's entropy, which measure randomness and peakyness of the signals. Given a scalar random variable $\mathrm{x}$, kurtosis is expressed as $k=m_{4}-3 m_{2}^{2}$, where $m_{\mathrm{n}}$ is the nth order central moment of the variable $m_{n}=E\left(x-m_{1}\right)^{n}$ and $m_{1}$ is the mean value. The kurtosis values are first normalized to zero mean and unit variance, and those with values larger than the threshold \pm 1.5 (the value is found by trial and errors) are selected as critical WCs. The recommended value of order for Renyi's entropy is 2 . WCs with entropy larger than the threshold \pm 1.5 after normalization are also selected as critical WCs. Fig. 4 shows an example of critical WC selection from the recording shown in Fig. 2. A total of 76 WCs are generated from this 19 electrode recording, following four frequency bands (delta, theta, alpha, and beta). With a threshold of \pm 1.5 , the red bars in Fig. 4 indicate the identified critical WCs. 
(3) Wavelet independent component (WIC) extraction. The selected critical WCs are then passed to ICA to separate the artifactual WICs. This system adopts the FastICA $2.5^{30}$ algorithm for Matlab. Fig. 5 shows an example of WICs obtained after application of ICA on the selected critical WCs. Artifacts with similar pattern as those annotated by the specialist in Fig. 2 can be identified independently.

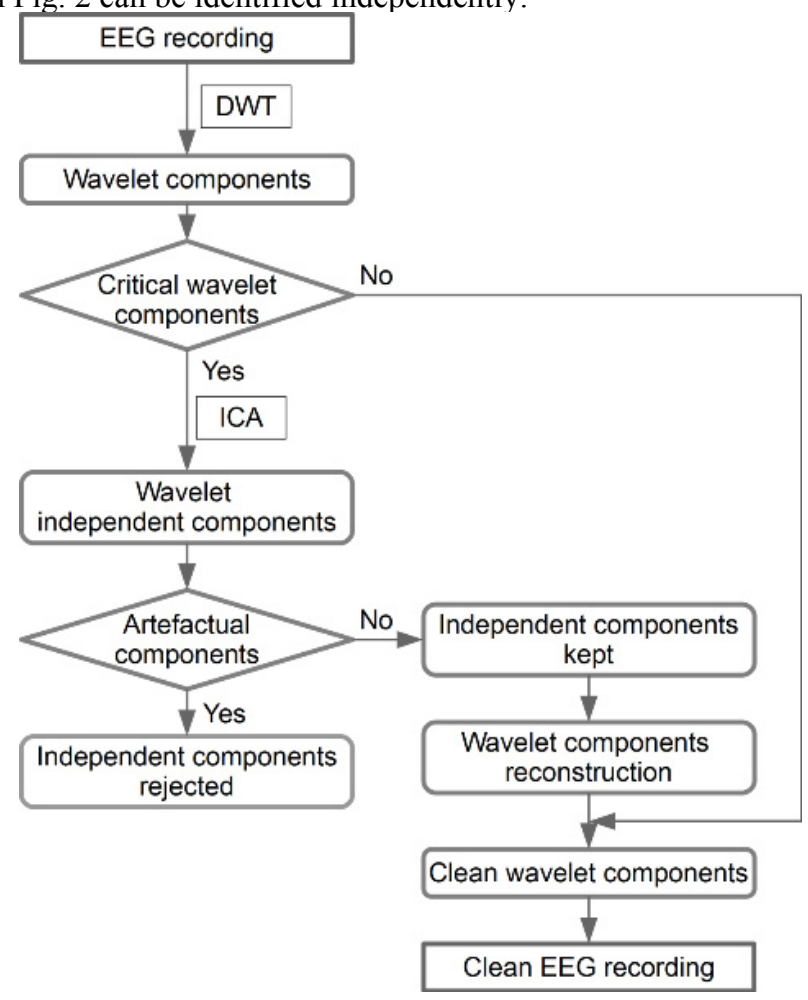

Fig. 3. AWICA artifacts removing algorithm (adapted ${ }^{21,25}$ )

(4) Artifactual WIC selection. This step concentrates on removing one or more WICs remained after using ICA. The selection of these artifactual WICs is also based on kurtosis and entropy. The difference in this step is that the WIC dataset is first divided into 0.5s non-overlapping windows (trials), and then the kurtosis and entropy are calculated based on these trials. WICs with more than $20 \%$ of the trial's kurtosis and entropy above the threshold \pm 1.5 are rejected.

(5) Reconstruction. The remaining WICs are then used to project back artifact-free WCs with an inverse ICA and combined with the non-critical WCs. The result is WCs cleaned from artifacts. Performing an inverse DWT enables the reconstruction of an artifact-free EEG recording.

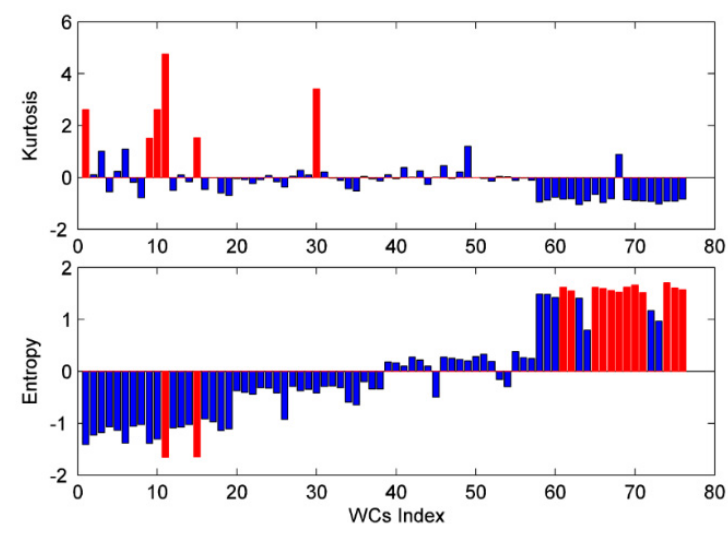


Fig. 4. Example of critical WC selection (threshold $= \pm 1.5$ ).

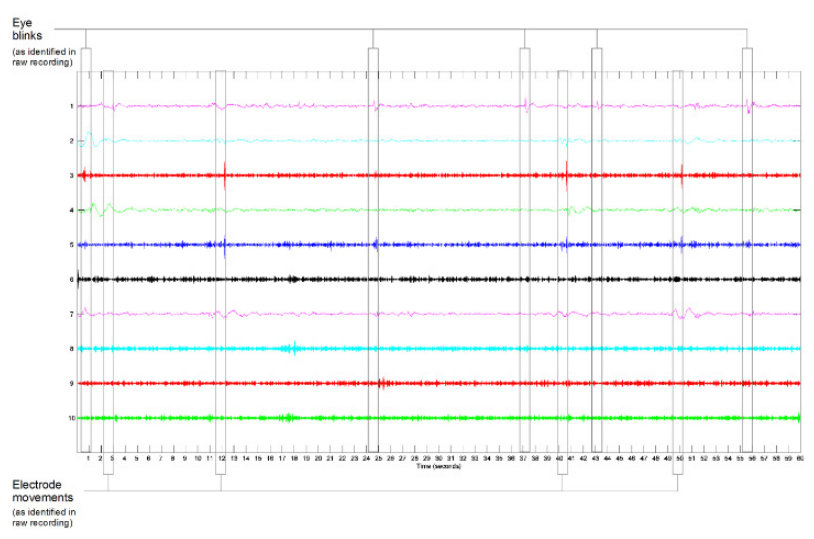

Fig. 5. Example of EEG data after application of ICA.

\section{4. qEEG Feature Extraction}

qEEG is a numerical analysis of EEG data using signal analysis techniques such as wavelet analysis and Fourier analysis. The commonly used features are EEG coherence, phase, power, and amplitude. The features are calculated based on artifact-free recordings for eight frequency bands: delta $(\delta, 1$ to $4 \mathrm{~Hz})$, theta $(\theta, 4$ to $8 \mathrm{~Hz})$, alpha $(\alpha, 8$ to 12 $\mathrm{Hz}$ ), beta ( $\beta, 8$ to $25 \mathrm{~Hz}$ ), hi-beta (hi- $\beta, 25$ to $30 \mathrm{~Hz}$ ), beta1 ( $\beta 1,12$ to $15 \mathrm{~Hz}$ ), beta2 ( $\beta 2,15$ to $18 \mathrm{~Hz}$ ), and beta3 $(\beta 3,18$ to $25 \mathrm{~Hz}$ ). The Fast Fourier Transform (FFT) converts signal $x$ from the time to the frequency domain:

$$
F(x)=f f t(x)
$$

The auto-spectrum AP at frequency $\mathrm{f}$ is:

$$
A P(x, f)=a(x, f)^{2}+b(x, f)^{2},
$$

where $a(x, f)$ and $b(x, f)$ are the cosine and sine coefficients at frequency $f$ for signal $x$. The amplitude A can then be obtained as the square root of the auto-spectrum: $A=\sqrt[2]{A P}$. The amplitude asymmetry $\mathrm{AA}^{31}$ for two signals $x$ and $y$ is calculated as

$$
A A(x, y, f)=\sqrt{\text { cospectrum }(f)^{2}+\text { quadspectrum }(f)^{2}},
$$

where cospectrum $=a(x, f) a(y, f)+b(x, f) b(y, f), \quad$ and quadspectrum $(f)=a(x, f) b(y, f)-$ $b(x, f) a(y, f)$.

Next, the coherence $\mathrm{CO}$ is computed for pair-wise combination of electrodes $\mathrm{x}$ and $\mathrm{y}$,

$$
C O(x, y, f)=\frac{C S(x, y, f)^{2}}{A P(x, f) A P(y, f)}=\frac{\text { cospectrum }(f)^{2}+\text { quadspectrum }(f)^{2}}{A P(x, f) A P(y, f)},
$$

The phase difference $\mathrm{PH}$ is then computed:

$$
P H=\operatorname{Arctan} \frac{\text { quadspectrum }(f)}{\operatorname{cospectrum}(f)} .
$$


These calculations allow the 16 features identified by Thatcher ${ }^{4}$ as discriminant in TBI diagnosis to be employed in the algorithm. The labels used in the features follow the 10-20 coding system used in EEG research and practice that indicate the position of the electrodes on the sculp. The letters F, T, C, P and O stand for frontal, temporal, central, parietal, and occipital lobes, respectively. Even numbers refer to electrode positions on the right hemisphere, whereas odd numbers denote those located on the left hemisphere. The number zero represents an electrode placed on the midline. The features employed in the research are the those selected by Thatcher ${ }^{3}$.

\subsection{Dataset Construction and Model Training}

When new field data is recorded, the TBI diagnosis is obtained by performing a classification prediction based on a comparison of the extracted vector of qEEG features defined above with the trained model. The discriminant analysis uses two classes: TBI and normal. The model is trained with the same qEEG features, extracted from EEG data previously recorded in a clinical setting. The dataset is composed of EEG data recorded from 21 electrodes (2 electrodes used as references) at a sample rate of $256 \mathrm{~Hz}$ using a BrainMaster device ${ }^{32}$. Recordings include data with patients' eyes open and closed. The recordings are annotated by specialists with labels corresponding to the International Statistical Classification of Diseases (ICD-10) system $^{33}$, thereby providing a ground truth for the classification.

The recordings used for training the model in this study have labels F07.2 and Avrg. The label F07.2 corresponds to a post-concussional syndrome, i.e., patients diagnosed with TBI. The label Avrg stands for average healthy subjects. In total, 288 recordings from 14 patients ( 8 female and 6 male) have been used, including 251 recordings labelled as F07.2 and 37 regarded as Avrg. After pre-processing and extraction of the discriminant features, the training dataset was constructed as an Nx16 matrix with $\mathrm{N}$ being the total number of clinical samples. A model with good generalization performance was obtained by splitting this dataset into a training dataset for model training and a validation dataset for evaluating the model.

As mentioned, the proposed method builds the model by performing a discriminant analysis. The relation between the selected qEEG features and the classes is assumed to follow a multivariate normal distribution. The mean of each feature is calculated for each class. The covariance is also calculated, after first subtracting the mean. Considering a linear discriminant analysis, the model has the same covariance for each class, only the means vary. No prior probabilities or costs are used to compute the model, as the labels define the class to which each sample belongs. The trained model is then used to predict the classification of newly acquired data. The principle is to find the class with the highest probability that the new sample belongs to. The obtained classification is then returned for each segment considering an online situation. This classification of multiple segments allows better precision to be achieved in the diagnosis.

\section{Evaluation}

This section evaluates the developed system in terms of its EEG processing and TBI diagnosis.

The proposed algorithm for TBI diagnosis has two main functions: pre-processing of EEG data, including an automatic artifact removal, and the diagnosis of TBI itself, based on discriminant qEEG features and the comparison with a model trained on previously collected and annotated data. The performance of the artifact-removing algorithm has a great impact on the final diagnosis due to the high signal-to-noise ratio. Thus, in this evaluation, the performance of the pre-processing algorithm is presented first, followed by the performance of the classification algorithm for TBI diagnosis.

\subsection{Evaluation of the pre-processing method}

For the purpose of evaluating the performance of the pre-processing algorithm in removing different types of artifacts, 20 randomly selected recordings were first visually inspected by a highly qualified specialist, who annotated each artifact with its type. In total, 225 artifacts of 6 different types were found by the specialist (Table I); these include eye blinking (138 instances), eye movement (48), electrode movement (14), muscle activity (3), 
drowsiness (3), and head movement (19). The dataset is representative as the total number of annotated eye blinks in these recordings is more than half of all marked artifacts, while the number of muscle activities and drowsiness periods is very low as these are easier to control and eliminate during the recordings.

Table 1. Artifact Annotation by a specialist and percentage of removal by the pre-processing algorithm

\begin{tabular}{llllllll}
\hline Type of artifact & Eye Blinking & Eye Movement & Electrode Movement & Muscle Activity & Drowsiness & Head Movement & Total \\
\hline Total & 138 & 48 & 14 & 3 & 3 & 19 & 225 \\
Removal \% & $84.8 \%$ & $83.3 \%$ & $85.7 \%$ & $0 \%$ & $66.7 \%$ & $36.8 \%$ & $79.1 \%$ \\
\hline
\end{tabular}

The artifact-removal algorithm was applied to the same 20 recordings. Table I shows the results. The results show that $79.1 \%$ of the annotated artifacts have been successfully removed. Most of the eye blinks (84.8\%), eye movements $(83.3 \%)$, and electrode movements $(85.7 \%)$ have been eliminated. These three groups represent the majority of artifacts in the dataset (88.88\%). The percentage of successful removal of artifacts related to drowsiness and head movement is lower, at $66.7 \%$ and $36.8 \%$, respectively. The algorithm has failed to remove any artifacts related to muscle activity; experiments with more data is needed to improve this parameter.

Fig. 6 shows signals obtained after pre-processing the raw segments shown in Fig. 2. The comparison between Fig. 6A, B and $\mathrm{C}$ shows that the success rate in removing artifacts depends on the threshold value. Small threshold values may result in rejection of some elements of the brain signals (see Fig. 6A, threshold \pm 1 ) while high threshold values may result in low success rate in artifact removal (Fig. 6C, threshold \pm 2 ). Fig. $6 \mathrm{~B}$ is obtained with the optimal threshold of \pm 1.5 .

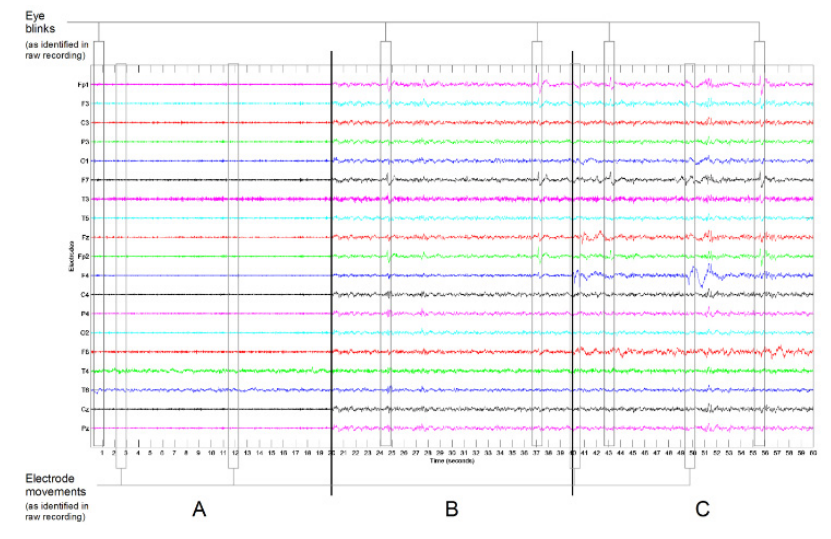

Fig. 6. Artifact-free epochs: A, threshold $= \pm 1 ; \mathrm{B}$, threshold $= \pm 1.5$; C, threshold $= \pm 2$

\section{Conclusion}

This paper proposes a method for an automatic early diagnosis of TBI in emergency situations. The system is based on state-of-the-art standards and advanced technologies for processing and intelligent diagnosis. The system has been specifically developed in response to real needs: fast and reliable assessment of possible brain injury where the accident occurred.

The development of the automatic TBI diagnosis algorithm is based on advanced EEG signal processing and machine learning techniques. The pre-processing step of the algorithm enables the automatic removal of artifacts and noise, avoiding the need for a time-consuming manual inspection and removal of data segments. The diagnosis is computed using supervised machine learning based on clinical data. The system operator is then provided with an assessment of the possible patient's traumatic brain injury.

The evaluation of the proposed algorithms has shown it to be fast and reliable, with a good generalization performance of the model. The result of the automatic diagnosis, coupled with a decision support within the EmerEEG system, provides the operator with an effective basis for the early application of an adapted treatment in an emergency situation.

Currently, the data stream from the head device is simulated with previously recorded data. The actual testing 
with humans is beyond the scope of this project. Future work includes integration of the head device with the portable system and clinical evaluations once medical approval is obtained.

\section{Acknowledgement}

The authors thank the European Commission for funding this research (Grant 605103, FP7-SME-2013). They also thank their partners Maytec, neuroConn, and University Hospital Gottingen from Germany, Primasil, UK and Tallinn University, Estonia.

\section{References}

1. Saatman K. E., Duhaime A., Bullock R., Maas A. I., Valadka A., Manley G., Classification of traumatic brain injury for targeted therapies, Neurotraum, vol. 25, no. 7, pp. 719-738, Nov. 2010.

2. Rimel R. W., Giordani B., Barth J. T., Boll T. J., Jane J. A., Disability caused by minor head injury, Neurosurgery, vol. 9, no. 3, pp. 221-228, Sep. 1981.

3. Thatcher R. W., Walker R. A., Gerson I., Geisler F. H., EEG discriminant analyses of mild head trauma, Electroenc. Clin. Neuro., vol. 73, no. 2, pp. 94-106, Aug. 1989.

4. Thatcher R. W., North D. M., Curtin R. T., Walker R. A., Biver C. J., Gomez J. F., Salazar A. M., An EEG severity index of traumatic brain injury, J. Neuropsych. Clin. N., vol. 13, no. 1, pp. 77-87, Feb. 2001.

5. Injury prevention and control: Traumatic brain injury - TBI data \& statistics, Centers for Disease Control and Prevention, Available: http://www.cdc.gov/traumaticbraininjury/data/index.html. [Accessed 21 July 2015].

6. Tagliaferri F., Compagnone C., Korsic M., Servadei F., Kraus J., A systematic review of brain injury epidemiology in Europe, Acta Neurochir., vol. 148, no. 3, pp. 255-268, Mar. 2006.

7. Gustavsson A., Svensson M., Jacobi F., Allgulander C., Alonso J., Beghi E., Dodel R., Ekman M., Faravelli C., Fratiglioni L., Gannon B., Jones D. H., Jennum P., Jordanova A., Cost of disorders of the brain in Europe 2010, Eur. Neuropsychopharmacol., vol. 21, no. 10, pp. 718-779, Oct. 2011.

8. Jennett G. T. B., Assessment of coma and impaired consciousness: a practical scale, Lancet, vol. 304, no. 7872, pp. 81-84, Jul. 1974.

9. Pal J., Brown R., Fleiszer D., The value of the Glasgow Coma Scale and Injury Severity Score: predicting outcome in multiple trauma patients with head injury, J. Trauma., vol. 29, no. 6, pp. 746-748, Jun. 1989.

10. Bigler E. D., Maxwell W. L., Neuroimaging and neuropathology of TBI, NeuroRehabilitation, vol. 28, no. 2, pp. 63-74, 2011.

11. Bardin J. C., Fins J. J., Katz D. I., Hersh J., Heier L. A., Tabelow K., Dyke J. P., Ballon D. J., Schiff N. D., Voss H. U., Dissociations between behavioural and functional magnetic resonance imaging-based evaluations of cognitive function after brain injury, Brain, vol. 134, no. 3, pp. 769782, Feb. 2011.

12. Lee B., Newberg A., Neuroimaging in traumatic brain imaging, NeuroRx, vol. 2, no. 2, pp. 372-383, Apr. 2005.

13. Thatcher R. W., Electroencephalography and mild traumatic brain injury, in Foundations of Sport-Related Brain Injuries, Springer, 2006, pp. 241-265.

14. Thatcher R. W., Cantor D. S., McAlaster R., Geisler F., Krause P., Comprehensive Predictions of Outcome in Closed Head-Injured Patients,. Ann. NY Acad. of Sci., vol. 620, no. 1, pp. 82-101, Apr. 1991.

15. Winkler I., Haufe S., Tangermann M., Automatic classification of artifactual ICA-components for artifact removal in EEG signals, Behavi. Brain Func., vol. 7, no. 1, p. 30, Aug. 2011.

16. Anderer P., Roberts S., Schlogl A., Gruber G., Klosch G., Herrmann W., Rappelsberger P., Filz O., Barbanoj M. J., Dorffner G., Saletu B., Artifact processing in computerized analysis of sleep EEG - a review, Neuropsychobiology, vol. 40, no. 3, pp. 150-157, Sep. 1999.

17. Fatourechi M., Bashashati A., Ward R. K., Birch G. E., EMG and EOG artefacts in brain computer interface systems: A survey, Clin. Neurophysiol., vol. 118, no. 3, pp. 480-494, Mar. 2007.

18. McFarland D. J., McCane L. M., David S. V., Wolpaw J. R., Spatial filter selection for EEG-based communication, Electroen. Clin. Neuro., vol. 103, no. 3, pp. 386-394, Sep. 1997.

19. Hyvarinen A., Oja E., Independent component analysis: algorithms and applications, Neural networks, vol. 13, no. 4, pp. 411-430, Jun. 2000. 20. Jung T.-P., Makeig S., Humphries C., Lee T.-W., Mckeown M. J., Iragui V., Sejnowski T. J., Removing electroencephalographic artifacts by blind source separation, Psychophysiology, vol. 37, no. 2, pp. 163-178, Mar. 2000.

21. Viola F. C., Thorne J., Edmonds B., Schneider T. E. T., Debener S., Semi-automatic identification of independent components representing EEG artifact, Clin. Neurophysiol., vol. 120, no. 5, pp. 868-877, May 2009.

22. Mognon A., Jovicich J., Bruzzone L., Buiatti M., ADJUST: An automatic EEG artifact detector based on the joint use of spatial and temporal features, Psychophysiology, vol. 48, no. 2, pp. 229-240, Feb. 2011.

23. LeVan P., Urrestarazu E., Gotman J., A system for automatic artifact removal in ictal scalp EEG based on independent component analysis and Bayesian classification, Clin. Neurophysiol., vol. 117, no. 4, pp. 912-927, Apr. 2006.

24. Halder S., Bensch M., Mellinger J., Bogdan M., Kubler A., Birbaumer N., Rosenstiel W., Online artifact removal for brain-computer interfaces using support vector machines and blind source separation, Comput. Intell. Neurosci., Apr. 2007.

25. Mammone N., La Foresta F., Morabito F. C., Automatic artifact rejection from multichannel scalp EEG by wavelet ICA, IEEE Sensors Journal, vol. 12, no. 3, pp. 533-542, Feb. 2012.

26. Castellanos N. P., Makarov V. A., Recovering EEG brain signals: artifact suppression with wavelet enhanced independent component analysis, J. Neurosci. Meth., vol. 158, no. 2, pp. 300-312, Dec. 2006.

27. Duffy F. H., Hughes J. R., Miranda F., Bernad P., Cook P., Status of quantitative EEG (QEEG) in clinical practice, Clin. EEG Neurosci., vol. 25 , no. 4, pp. 6-22, Oct. 1994. 
28. Hughes J. R., John E. R., Conventional and quantitative electroencephalography in psychiatry, J. Neuropsych. Clin. N., vol. 11, no. 2, pp. 190-208, May 1999.

29. European Data Format, [Online]. Available: http://www.edfplus.info/. [Accessed 21 July. 2015].

30. Gavert H., Hurri J., Sarela J., Hyvarinen A., Fastica 2.5, [Online]. Available: http://research.ics.aalto.fi/ica/fastica/. [Accessed 12 Jan. 2015].

31. Neuroguide help manual, Applied Neuroscience, [Online]. Available: http://www. appliedneuroscience.com/Tutorials.htm. [Accessed 12 Jan. 2015].

32. Brainmaster Technologies, Inc., [Online]. Available: http://www.brainmaster.com/. [Accessed 21 July 2015].

33. International statistical classification of diseases and related health problems 10th revision, World Health Organization, [Online]. Available: http://apps.who.int/classifications/icd10/browse/2015/en. [Accessed Jan. 2015] 